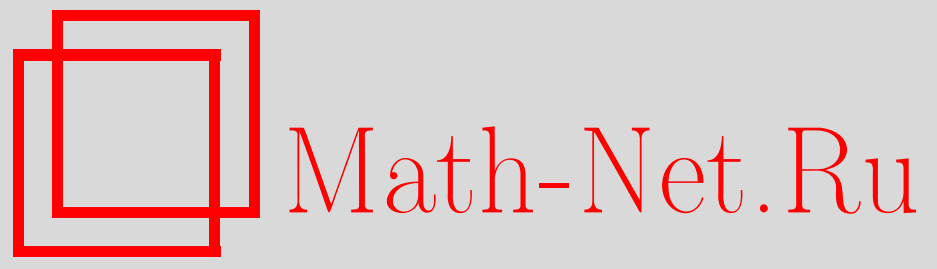

W. Jedidi, Stable Processes, Mixing, and Distributional Properties. I, Теория вероятн. и ее примен., 2007, том 52, выпуск 4, 736-751

DOI: https://doi.org/10.4213/tvp1531

Использование Общероссийского математического портала Math-Net.Ru подразумевает, что вы прочитали и согласны с пользовательским соглашением

http://www . mathnet.ru/rus/agreement

Параметры загрузки:

IP : 54.198 .67 .100

26 апреля 2023 г., 10:54:34

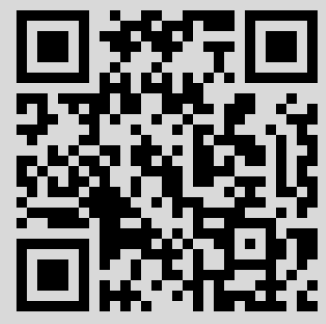




\section{STABLE PROCESSES, MIXING AND DISTRIBUTIONAL PROPERTIES. I}

В статье рассматриваются действительнозначные устойчивые процессы Леви $\left(S_{t}^{\alpha, \beta, \gamma, \delta}\right)_{t \geqslant 0}$, где $\alpha, \beta, \gamma, \delta$ - коэффициенты устойчивости, асимметрии, масштаба и сноса соответственно. Вводится понятие смешанных устойчивых процессов $\left(M_{t}^{\alpha, \beta, \gamma, \delta}\right)_{t \geqslant 0}$ (для которых последние три коэффициента могут быть случайными). Наша смешивающая процедура приводит к структуре условных процессов Леви. Эта процедура позволяет показать, что сумма независимых устойчивых процессов может быть выражена через смешанный устойчивый процесс.

Ключевые слова и фразы: устойчивые процессы, процессы с условно независимыми стационарными приращениями, свертка Меллина, плотность, производные.

\section{Introduction}

Problem of small time for densities of Lévy processes is an important issue in the framework of the statistic of Lévy processes. For example, consider the scale model associated with the observations at times $i / n, 1 \leqslant i \leqslant n$, of the product $X=\theta Y$, where $\theta$ is an unknown parameter and $Y$ is a known Lévy process having at each time $t>0$ a density function $g_{t}$. Statistical properties like local asymptotic normality of the associated sequence of models are required. At first glance, things seem to be trivial, but they start to be intricate when, for example, one seeks when the asymptotic Fisher information quantity is finite nonnegative. One has to deal with the following related limit:

$$
I=\lim _{t \rightarrow 0} \int\left(1+x \bar{g}_{t}(x)\right)^{2} g_{t}(x) \mathrm{d} x
$$

where $\bar{g}_{t}$ is the derivative of $x \mapsto \ln g_{t}(x)$. Notice that the density $g_{t}$, if it exists and is differentiable, is nonexplicit in general and degenerates when $t \rightarrow 0$. Intuitively, one looks at a special class of Lévy processes $Y$ satisfying:

* LPMA CNRS-UMR 7599, Université P. et M. Curie (Paris 6); Department of Mathematics, Johannes Gutenberg University Mainz; Staudingerweg 9, D-55099 Mainz; e-mail: wissem_jedidi@yahoo.fr 
there exist measurable functions $b(t) \in \mathbf{R}, a(t)>0$ and a nondegenerate law $\nu$ such that the convergence in distribution holds:

$$
\tilde{Y}_{t}=\frac{Y_{t}-b(t)}{a(t)} \longrightarrow \nu, \quad t \rightarrow 0 \text { or } \infty .
$$

A known result (see [1] for $t \downarrow 0$ ) says that the process $Y$ is attracted by a strict stable distribution. More precisely, if the convergence holds, then necessarily $b(t)=b t, a(t)=t^{1 / \alpha} l(t)$ (where $b \in \mathbf{R}, \alpha \in(0,2]$, and $l(t)$ is a slowly varying function, i.e., $l(\lambda t) / l(t) \rightarrow 1$ for all $\lambda>0)$ and the convergence of $\widetilde{Y}_{t}$ can be entirely expressed with the behavior of the tail of the Lévy measure of $Y$ (case $\alpha<2$ ) or by the existence of a Brownian component in $Y$ (case $\alpha=2, l(t)$ constant). Moreover, for all $t>0, \tilde{Y}_{t}$ admits an infinitely differentiable probability density $H_{t}$ which satisfies for all $k \in \mathbf{N}$

$$
\left(H_{t}\right)^{(k)}(x) \rightarrow G^{(k)}(x), \quad t \rightarrow 0,
$$

uniformly in $x \in \operatorname{supp}(G), G$ being the density function of the stable distribution. If $\bar{H}_{t}$ (respectively, $x \mapsto \bar{G}$ ) denotes the derivative of $x \mapsto \ln H_{t}(x)$ (respectively, $\ln G$ ), then, after the suitable change of variable, $I$ should be equal to $\lim _{t \rightarrow 0} \int\left[1+\left(x+b(t) a(t)^{-1} \bar{H}_{t}(x)\right]^{2} H_{t}(x) \mathrm{d} x\right.$. Of course, the convergences (1.2) for all $k \in \mathbf{N}$ not sufficient to prove $I \in(0, \infty)$, but at least they ensure that if it is true, then

$$
\begin{gathered}
I=J \quad \text { with } \\
J=\int[1+(x+l) \bar{G}(x)]^{2} G(x) \mathrm{d} x \quad \text { and } \quad l=\lim _{t \rightarrow 0} b(t) a(t)^{-1} \in \mathbf{R}
\end{gathered}
$$

and the last integral is shown - thanks to Theorem 1 of Part II of this paper (to be published in a forthcoming issue of the journal) to be in $(0,+\infty)$. Actually, what one expects is to have more than (1.2). A control of the type $\lim \sup _{|x| \rightarrow \infty} \sup _{t<T}\left|x \bar{H}_{t}(x)\right|<\infty$ would be sufficient to prove that $I=J$. Unfortunately, here also, there is a lack in the literature concerning such controls. Some authors such that Ishikawa [4] (using Malliavin calculus) or Picard [8], show results concerning controls in small time for densities of jump processes. Their results are either global in the space variable, or simple in time, and then we cannot use them in our framework. Nevertheless, in Part II of this paper, we provide examples of Lévy processes of the last kind. For this purpose we introduce in Section 3 the notion of mixed stable processes $\left(M_{t}^{\alpha, \beta, \gamma, \delta}\right)_{t \geqslant 0}$, on the skewness, scale, and drift coefficients, i.e., we allow $(\beta, \gamma, \delta)$ to be random. Our mixing procedure give to the processes $\left(M_{t}^{\alpha, \beta, \gamma, \delta}\right)_{t \geq 0}$ a structure of conditional-Lévy processes. These processes are natural extensions of the proper stable process and actually inherit from them many properties like (like scaling property). In some cases, mixed stable processes are more flexible than proper stable processes. For example, 
in many situations, the sums of independent stable processes with different stability coefficients have the same finite-dimensional distribution as mixed stable variable:

$$
\sum_{k=1}^{n} S_{t}^{a_{k}, b_{k}, c_{k}, d_{k}} \stackrel{d}{=} M_{1}^{\alpha, \beta_{t}, \gamma_{t}, \delta_{t}}
$$

where the deterministic index of stability $\alpha$ and the random triplet $\left(\beta_{t}, \gamma_{t}, \delta_{t}\right)$ are build explicitly from the quartets $\left(a_{k}, b_{k}, c_{k}, d_{k}\right)_{k=1, \ldots, n}$.

Many of these examples and tools were initiated in [6] by statistical motivations.

\section{Description of stable processes}

Let $\left(\Omega, \mathscr{D},\left(\mathscr{F}_{t}\right)_{t \geqslant 0}, \mathbf{P}\right)$ be a stochastic basis, $A=(0,2] \times[-1,1] \times$ $(0,+\infty) \times \mathbf{R}$

$$
K(\alpha)=\alpha \mathbb{1}_{(0,1]}(\alpha)+(\alpha-2) \mathbb{1}_{(1,2]}(\alpha), \quad \text { and } \quad \bar{K}(\alpha)=K(\alpha) \alpha^{-1} .
$$

Let $(\alpha, \beta, \gamma, \delta) \in A$. Adopting Zolotarev's conventions [11, p. 12], we say that under $\mathbf{P}$, a real-valued process denoted by $S^{\alpha, \beta, \gamma, \delta}$ is a stable process, if it is a Lévy process, and if for all $t \geqslant 0$ and $u \in \mathbf{R}$ its characteristic functions are defined by $\mathbb{E}\left[\exp \left\{\mathrm{i} u S_{t}^{\alpha, \beta, \gamma, \delta}\right\}\right]=\exp \left\{t \varphi^{\alpha, \beta, \gamma, \delta}(u)\right\}$, where

$$
\varphi^{\alpha, \beta, \gamma, \delta}(u)= \begin{cases}\mathrm{i} \delta u-\gamma|u|^{\alpha} \exp \left(-\frac{\mathrm{i} \pi \beta}{2} K(\alpha) \operatorname{sign}(u)\right) & \text { if } \quad \alpha \neq 1, \\ \mathrm{i} \delta u-\gamma|u|\left(1+\frac{\mathrm{i} 2 \beta}{\pi} \ln |u| \operatorname{sign}(u)\right) & \text { if } \quad \alpha=1 .\end{cases}
$$

The constants $\alpha, \beta, \gamma, \delta$ are respectively called coefficient (or index) of stability, skewness, scale, and drift of the process $S^{\alpha, \beta, \gamma, \delta}$. P. Lévy [7] in 1924 was the first to introduce stable laws known under their current form. His main objective was to characterize the random variables $Z$ satisfying: for $n \geqslant 2$ there exist $a_{n}>0, b_{n} \in \mathbf{R}$ such that $\sum_{i=1}^{n} Z_{i}$ has the same law as $a_{n} Z+b_{n}$, where $Z_{i}$ are independent copies of $Z$. He showed that the variables $S_{1}^{\alpha, \beta, \gamma, \delta}$, with $\beta=0$ if $\alpha=1$, satisfy the previous property and that necessarily $a_{n}=n^{1 / \alpha}, b_{n}=b n, b \in \mathbf{R}$. The following scaling property is true for all $t>0$, and with $\delta(\alpha, t)=t\left[\delta+(2 \beta / \pi) \gamma \ln (\gamma t) \mathbb{1}_{\{\alpha=1\}}\right]$, we have

$$
S_{t}^{\alpha, \beta, \gamma, \delta} \stackrel{d}{=} S_{1}^{\alpha, \beta, \gamma t, \delta t} \stackrel{d}{=} S_{\gamma t}^{\alpha, \beta, 1, \delta / \gamma} \stackrel{d}{=} \delta(\alpha, t)+(\gamma t)^{1 / \alpha} S_{1}^{\alpha, \beta, 1,0} .
$$

The appearance of stable processes in the most known limit theorems justified the efforts dedicated by Gnedenko and Kolmogorov [3] and Feller [2] (amongst others) to the study of these processes. Zolotarev [11] has written a book on stable laws, on which we rely heavily. Moreover, stable processes are the prototype of the Lévy processes, entirely characterized by their Lévy exponents. 
2.1. Some classes of stable processes. Intuitively, the coefficients $\alpha, \beta, \gamma$, and $\delta$ are arranged according to the decreasing «impact» on the process $S^{\alpha, \beta, \gamma, \delta}$. This fact clears little by little through this paper. Notice that in the characterization $(2.2), K(2)=0$ and the law of $S^{2, \beta, \gamma, \delta}$ does not depend on $\beta$ and in fact $S_{t}^{2, \beta, \gamma, \delta}$ is equal to $\sqrt{2 \gamma} W_{t}+\delta t$, where $W$ is a standard Brownian motion. It is then reasonable to put $\beta=0$ when $\alpha=2$ in order to emphasize on the symmetry of the processes $S^{2, \beta, \gamma, 0}$. Notice also that the case $\alpha=1, \beta \neq 0$ is singular when mixing stable processes, and it turns out to be intricate. Then, we introduce some subsets of $A$ and the corresponding classes of law of processes:

$$
\begin{aligned}
B & =\{(\alpha, \beta, \gamma, \delta) \in A, \beta=0 \text { if } \alpha=2\}, \\
C & =\{(\alpha, \beta, \gamma, \delta) \in B, \beta=0 \text { if } \alpha=1\}, \\
C_{\mathrm{drf}} & =\{(\alpha, \beta, \gamma, \delta) \in C, \delta=0 \text { if } \alpha \neq 1\}, \\
C_{\text {skw }} & =\left\{(\alpha, \beta, \gamma, 0) \in C_{\mathrm{drf}},|\beta|<1 \text { if } \alpha \geqslant 1\right\}, \\
S_{\text {ext }} & =\{(\alpha, \beta, \gamma, \delta) \in B: \beta=-1,1\}, \\
S_{\text {ubo }} & =\left\{(\alpha, 1, \gamma, \delta) \in S_{\text {ext }}: \alpha \leqslant 1, \delta \geqslant 0\right\} .
\end{aligned}
$$

For $E \subseteq A$, when we write $S^{\alpha, \beta, \gamma, \delta} \in \mathscr{C} \mathscr{L}(E)$, it is understood that un$\operatorname{der} \mathbf{P}, S^{\alpha, \beta, \gamma, \delta}$ is a stable process such that the quartet $(\alpha, \beta, \gamma, \delta)$ is in $E$. The processes $S^{1,0, \gamma, \delta} \in \mathscr{C} \mathscr{L}\left(C_{\text {drf }}\right)$ are $\delta$-drifted Cauchy processes and it is a well-known fact that $\mathscr{C} \mathscr{L}\left(S_{\text {ext }}\right)$ is the class of extreme stable processes (i.e., with unilateral jumps, no jumps for $\alpha=2), \mathscr{C} \mathscr{L}\left(S_{u b o}\right)$ being the class of stable subordinators (see Appendix). When we write $S^{1,1, \gamma, \delta} \in \mathscr{C} \mathscr{L}\left(S_{\text {ubo }}\right)$, that denotes $(\gamma+\delta)$ times the identity process, and in fact for each $t \geqslant 0$, the random variable $S_{t}^{1,1, \gamma, \delta}$ is considered as the limit in distribution of $S_{t}^{\alpha, 1, \gamma, \delta}$ as $\alpha \nearrow 1$. Because of the scaling property (2.3) and in order to simplify the expressions, we adopt the following notation: if the coefficients $\beta, \gamma$ or $\delta$ are omitted, that would mean that $\beta=1, \gamma=1$, and $\delta=0$ and we will denote

$$
\begin{gathered}
S^{\alpha, \beta, \gamma}=S^{\alpha, \beta, \gamma, 0}, \quad S^{\alpha, \beta}=S^{\alpha, \beta, 1}, \quad S^{\alpha}=S^{\alpha, 1}, \quad \text { and } \\
S^{1}=\operatorname{Identity}\left(\mathscr{C} \mathscr{L}\left(S_{u b o}\right)\right) .
\end{gathered}
$$

\section{The sum of independent stable processes is a mixed stable process}

We have the convention: $\frac{0}{0}=0$. If $U$ and $V$ are two r.v. (respectively, processes), then $U \stackrel{d}{=} V$ (respectively, $U \stackrel{\mathscr{L}}{=} V$ ) means that they have the same law. When $V_{t} \geqslant 0, U \circ V_{t}$ denotes a process $U$ taken at time $V_{t}$. 
3.1. The subordination relation. When $(a, b, c, d) \in C_{\mathrm{drf}}$, we define

$$
\begin{aligned}
& \theta(a, b, c, d):= \begin{cases}b \bar{K}(a) & \text { if } a \neq 1, \\
\frac{2}{\pi} \arcsin \frac{d}{\sqrt{c^{2}+d^{2}}} & \text { if } a=1,\end{cases} \\
& \rho(a, b, c, d):=\frac{1+\theta(a, b, c, d)}{2} .
\end{aligned}
$$

Notice, according to (4.5), that $\rho(a, b, c, d)=\mathbf{P}\left\{S_{t}^{a, b, c, d}>0\right\}$ for all $t>0$. The following proposition is a first result which gives a taste of those obtained in this subsection.

Proposition 1. Let $S^{\alpha, \beta}, S^{\alpha^{\prime}, \beta^{\prime}} \in \mathscr{C} \mathscr{L}(C), \alpha \geqslant \alpha^{\prime}$, and let $S^{\alpha^{\prime} / \alpha} \in$ $\mathscr{C} \mathscr{L}\left(S_{u b o}\right)$ be independent from $S^{\alpha, \beta}$. Then

$$
S^{\alpha^{\prime}, \beta^{\prime}} \stackrel{\mathscr{L}}{=} S^{\alpha, \beta} \circ S^{\alpha^{\prime} / \alpha} \quad \text { if and only if } \mathbf{P}\left\{S_{1}^{\alpha, \beta}>0\right\}=\mathbf{P}\left\{S_{1}^{\alpha^{\prime}, \beta^{\prime}}>0\right\} .
$$

Now, we will extend this result by taking nonstandard scale and drift parameters in the previous processes. We need then to characterize when the equality $\theta(\alpha, \beta, \gamma, \delta)=\theta\left(\alpha^{\prime}, \beta^{\prime}, \gamma^{\prime}, \delta^{\prime}\right)$ holds. The following definition clarifies this fact.

Def in ition 1. Recall that $\bar{K}$ is defined in (2.1).

(i) Let $(\alpha, \beta, \gamma, \delta) \in B$. We define $D_{\alpha, \beta, \gamma, \delta}^{\downarrow}$ as the set containing $(\alpha, \beta, \gamma, \delta)$ and containing, only in the case $(\alpha, \beta, \gamma, \delta) \in C_{\mathrm{drf}}$, the quartets $\left(\alpha^{\prime}, \beta^{\prime}, \gamma^{\prime}, \delta^{\prime}\right) \in C_{\text {skw }}$ such that

$$
0<\alpha^{\prime}<\alpha, \quad \beta^{\prime}=\frac{\theta(\alpha, \beta, \gamma, \delta)}{\bar{K}\left(\alpha^{\prime}\right)}, \quad \gamma^{\prime}=\left(\gamma^{2}+\delta^{2}\right)^{\alpha^{\prime} /(2 \alpha)}, \quad \text { and } \quad \delta^{\prime}=0 .
$$

(ii) Let $\left(\alpha^{\prime}, \beta^{\prime}, \gamma^{\prime}, \delta^{\prime}\right) \in B$. We define

$$
D\left(\alpha^{\prime}, \beta^{\prime}\right)= \begin{cases}{\left[\alpha^{\prime}, 1\right)} & \text { if } \quad\left|\beta^{\prime}\right|=1 \text { and } \alpha^{\prime}<1, \\ \left\{\alpha^{\prime}\right\} & \text { if }\left|\beta^{\prime}\right|=1 \text { and } 1 \leqslant \alpha^{\prime}<2, \\ {\left[\alpha^{\prime}, 2\left(1+\left|\beta^{\prime} \bar{K}\left(\alpha^{\prime}\right)\right|\right)^{-1}\right]} & \text { if } \quad\left|\beta^{\prime}\right|<1,\end{cases}
$$

and let $D_{\alpha^{\prime}, \beta^{\prime}, \gamma^{\prime}, \delta^{\prime}}^{\dagger}$ be the set containing $\left(\alpha^{\prime}, \beta^{\prime}, \gamma^{\prime}, \delta^{\prime}\right)$ and containing, only in the case $\delta^{\prime}=0$, the quartets $(\alpha, \beta, \gamma, \delta) \in C_{\mathrm{drf}}$ such that

$$
\begin{gathered}
\alpha \in D\left(\alpha^{\prime}, \beta^{\prime}\right), \quad \beta=\mathbb{1}_{\{\alpha \neq 1\}} \beta^{\prime} \frac{\bar{K}\left(\alpha^{\prime}\right)}{\overline{\bar{K}}(\alpha)}, \quad \text { and } \\
\gamma+\mathrm{i} \delta=\gamma^{\alpha / \alpha^{\prime}} \exp \left\{\mathrm{i} \frac{\pi}{2} \beta^{\prime} \mathbb{1}_{\{\alpha=1\}}\right\} .
\end{gathered}
$$

We denote by $\mathscr{C} \mathscr{L}\left(D_{\alpha^{\prime}, \beta^{\prime}, \gamma^{\prime}, \delta^{\prime}}^{\dagger}\right)$ the class of subordinand processes of $S^{\alpha^{\prime}, \beta^{\prime}, \gamma^{\prime}, \delta^{\prime}}$ and we write

$$
S^{\alpha^{\prime}, \beta^{\prime}, \gamma^{\prime}, \delta^{\prime}} \preceq S^{\alpha, \beta, \gamma, \delta} \quad \text { and } \quad S^{\alpha^{\prime}, \beta^{\prime}, \gamma^{\prime}, \delta^{\prime}} \prec S^{\alpha, \beta, \gamma, \delta} \quad \text { if } \quad \alpha^{\prime}<\alpha .
$$


$\mathrm{Re} \mathrm{m}$ a rk 1. Notice first that in (i) and (ii), $\theta(\alpha, \beta, \gamma, \delta)=$ $\theta\left(\alpha^{\prime}, \beta^{\prime}, \gamma^{\prime}, \delta^{\prime}\right)$ always holds and $\alpha=\alpha^{\prime}$ if and only if $(\alpha, \beta, \gamma, \delta)=$ $\left(\alpha^{\prime}, \beta^{\prime}, \gamma^{\prime}, \delta^{\prime}\right)$. Otherwise, necessarily $\left(\alpha^{\prime}, \beta^{\prime}, \gamma^{\prime}, \delta^{\prime}\right) \in C_{\text {skw }}$, and $(\alpha, \beta, \gamma, \delta) \in$ $C_{\mathrm{drf}}$. The function $a \mapsto|\bar{K}(a)|$ being decreasing, we assumed when $\left|\beta^{\prime}\right|<1$, that $\alpha \leqslant 2 /\left(1+\left|\beta^{\prime} \bar{K}\left(\alpha^{\prime}\right)\right|\right)$, which is equivalent to $|\beta|=\left|\beta^{\prime} \bar{K}\left(\alpha^{\prime}\right) / \bar{K}(\alpha)\right| \leqslant 1$ and this condition ensures that $D_{\alpha^{\prime}, \beta^{\prime}, \gamma^{\prime}, \delta^{\prime}}^{\uparrow} \subset C_{\mathrm{drf}}$. Finally, if $\alpha^{\prime}<2$ and $\left(\alpha^{\prime}, \beta^{\prime}, \gamma^{\prime}, \delta^{\prime}\right) \in C_{\text {skw }}$, then $\mathscr{C} \mathscr{L}\left(D_{\alpha^{\prime}, \beta^{\prime}, \gamma^{\prime}, \delta^{\prime}}\right)$ always contains, when $\alpha^{\prime}<\alpha<2 /\left(1+\left|\beta^{\prime} \bar{K}\left(\alpha^{\prime}\right)\right|\right)$, a process $S^{\alpha, \beta, \gamma, \delta}$. The subordination relation is a relation of order on $\mathscr{C} \mathscr{L}(B)$ (obviously partial) which keeps some special properties of the processes. More precisely, if $S^{\alpha^{\prime}, \beta^{\prime}, \gamma^{\prime}, \delta^{\prime}} \preceq S^{\alpha, \beta, \gamma, \delta}$, then $S^{\alpha^{\prime}, \beta^{\prime}, \gamma^{\prime}, \delta^{\prime}}$ is symmetrical (respectively, subordinator or opposite of a subordinator) if and only if $S^{\alpha, \beta, \gamma, \delta}$ has the same property. However, an extreme element $S^{\alpha, 1, \gamma}$ of $\mathscr{C} \mathscr{L}\left(S_{\text {ext }}\right), \alpha \in(1,2)$, is a subordinand of nonextreme elements of $\mathscr{C} \mathscr{L}\left(C_{\text {skw }}\right)$.

Now, we will present the motivation for introducing the subordination relation in the following theorem, and essentially in Theorem 3 below.

Theorem 1. Assume that the processes involved in each part of the following identities are independent.

(i) Let $S^{\alpha, \beta, \gamma, \delta} \in \mathscr{C} \mathscr{L}\left(C_{\mathrm{drf}}\right)$ and $S^{\varepsilon, 1,1, d} \in \mathscr{C} \mathscr{L}\left(S_{u b o}\right)$. Then

$$
S^{\alpha, \beta, \gamma, \delta} \circ S^{\varepsilon, 1,1, d} \stackrel{\mathscr{L}}{=} d^{1 / \alpha} S^{\alpha, \beta, \gamma, \delta}+S^{\alpha^{\prime}, \beta^{\prime}, \gamma^{\prime}},
$$

where $\alpha^{\prime}=\varepsilon \alpha$ and $S^{\alpha^{\prime}, \beta^{\prime}, \gamma^{\prime}} \in \mathscr{C} \mathscr{L}\left(D_{\alpha, \beta, \gamma, \delta}^{\downarrow}\right)$.

Then

(ii) Let $S^{\alpha^{\prime}, \beta^{\prime}, \gamma^{\prime}, \delta^{\prime}} \in \mathscr{C} \mathscr{L}(B), S^{\alpha, \beta, \gamma, \delta} \in \mathscr{C} \mathscr{L}\left(D_{\alpha^{\prime}, \beta^{\prime}, \gamma^{\prime}, \delta}^{\uparrow}\right)$, and $d \geqslant 0$.

$$
S^{\alpha^{\prime}, \beta^{\prime}, \gamma^{\prime}, \delta^{\prime}}+d^{1 / \alpha} S^{\alpha, \beta, \gamma, \delta} \stackrel{\mathscr{L}}{=} S^{\alpha, \beta, \gamma, \delta} \circ S^{\varepsilon, 1,1, d},
$$

where $\varepsilon=\alpha^{\prime} / \alpha$ and $S^{\varepsilon, 1,1, d} \in \mathscr{C} \mathscr{L}\left(S_{u b o}\right)$.

$\mathrm{R}$ e $\mathrm{m}$ a r k 2. This theorem generalizes the identities 3.3 .1 and 3.3 .10 in [11], which are the analogs of (3.2) expressed with $d=0$ and at fixed times $t>0$. Notice that (3.3) is more interesting than (3.2) in the sense that it collects within $\mathscr{C} \mathscr{L}\left(C_{\mathrm{drf}}\right)$ all the subordinand processes of a fixed process in $\mathscr{C} \mathscr{L}\left(C_{\text {skw }}\right)$. Moreover, notice that (3.3) is relevant only if $\alpha>\alpha^{\prime}$ and this is possible only if $S^{\alpha^{\prime}, \beta^{\prime}, \gamma^{\prime}, \delta^{\prime}} \in \mathscr{C} \mathscr{L}\left(C_{\text {skw }}\right)$. Notice that $\alpha=\alpha^{\prime}$ if and only if $\beta=b, \gamma=\gamma^{\prime}, \delta=\delta^{\prime}, \varepsilon=1$, and according to our notation, $S^{1,1,1, d}$ is understood to be $(1+d)$ times the identity process so that everything becomes trivial in (3.2) and (3.3). Notice also that this theorem can be generalized to the sum of more than two independent stable processes.

Pro of of Proposition 1. Just take $\gamma=\gamma^{\prime}=1$ and $d=\delta=0$ in Theorem 1.

Corollary 1. Let $S^{\alpha^{\prime}, \beta^{\prime}, \gamma^{\prime}} \in \mathscr{C} \mathscr{L}\left(C_{\text {skw }}\right)$ and $S^{\alpha, \beta, \gamma, \delta} \in \mathscr{C} \mathscr{L}\left(D_{\alpha^{\prime}, \beta^{\prime}, \gamma^{\prime}}^{\uparrow}\right)$ be independent. For all $d \geqslant 0$ and $t>0$, with $\rho$ defined in (3.1), one has $\mathbf{P}\left\{S_{t}^{\alpha^{\prime}, \beta^{\prime}, \gamma^{\prime}}+d S_{t}^{\alpha, \beta, \gamma, \delta}>0\right\}=\mathbf{P}\left\{S_{1}^{\alpha, \beta, \gamma, \delta}>0\right\}=\rho(\alpha, \beta, \gamma, \delta)=\rho\left(\alpha^{\prime}, \beta^{\prime}, 1,0\right)$. 
P r o o f. It is a simple application of (3.3), the scaling property (2.3), the positivity of $S^{\alpha^{\prime} / \alpha, 1,1, d^{\alpha}}$, and (4.5).

Before proving Theorem 1, we need a result due to Zolotarev [10], which says that a Lévy process time changed by an independent subordinator is also a Lévy process. This result is showed in the book of Sato [9] in a more complete form and gives explicitly all the characteristics of the time changed Lévy process.

Theorem 2. Let $h$ be a truncation function, $X$ a Lévy process with characteristics $\left(d^{X}, c^{X}, \nu^{X}\right)$ with respect to $h$, and $\eta$ an independent subordinator with Lévy exponent

$$
\ln \mathbb{E}\left[\exp \left\{\mathrm{i} u \eta_{1}\right\}\right]=\mathrm{i} u D^{\eta}+\int_{(0, \infty)}\left(e^{\mathrm{i} u t}-1\right) \nu^{\eta}(\mathrm{d} t), \quad D^{\eta} \geqslant 0 .
$$

Define the process $Y=X \circ \eta$. Then $Y$ is a Lévy process and its characteristics with respect to $h$ are $\left(d^{Y}, c^{Y}, \nu^{Y}\right)=D^{\eta}\left(d^{X}, c^{X}, \nu^{X}\right)+\left(d^{\eta, X}, 0, \nu^{\eta, X}\right)$, where for all Borelian set $\mathscr{B} \subset \mathbf{R}^{\star}$

$$
d^{\eta, X}=\int_{(0, \infty)} \mathbb{E}\left[h\left(X_{t}\right)\right] \nu^{\eta}(\mathrm{d} t) \quad \text { and } \quad \nu^{\eta, X}(\mathscr{B})=\int_{(0, \infty)} \mathbf{P}\left\{X_{t} \in \mathscr{B}\right\} \nu^{\eta}(\mathrm{d} t) .
$$

R e m a r k 3. The characteristics $\left(d^{Y}, c^{Y}, \nu^{Y}\right)$ are calculated in [9] only for $h(x)=x \mathbb{1}_{\{|x| \leqslant 1\}}$ and it is easy to see that the result remains true for any truncation function $h$.

P r o of of The or e m 1. We treat the nontrivial case $\alpha^{\prime}<\alpha$. Fix the truncation function $h(x)=\sin x$ and apply Theorem 2 with $X:=$ $S^{\alpha, \beta, \gamma, \delta} \in \mathscr{C} \mathscr{L}\left(C_{\mathrm{drf}}\right)$ independent from $\eta:=S^{\varepsilon, 1,1, d} \in \mathscr{C} \mathscr{L}\left(S_{u b o}\right)$. One has $D^{\eta}=d$ and $\nu^{\eta}=\lambda_{0}(\varepsilon, 1,1) t^{-\varepsilon-1} \mathbb{1}_{\{t>0\}} \mathrm{d} t$, with $\lambda_{0}(\varepsilon, 1,1)$ defined in (4.4) and equal to $\varepsilon / \Gamma(1-\varepsilon)$. Let us express $d^{\eta, X}$ and $\nu^{\eta, X}$.

1) Notice first, that for any $S^{\alpha, \beta, \gamma, \delta}$ in $\mathscr{C} \mathscr{L}(B)$, one has $\mathbb{E}\left[\sin S_{t}^{\alpha, \beta, \gamma, \delta}\right]=$ $\operatorname{Im} \mathbb{E}\left[\exp \left\{\mathrm{i} S_{t}^{\alpha, \beta, \gamma, \delta}\right\}\right]=\operatorname{Im} \exp \left\{t\left[\mathrm{i} \delta-\gamma\left(\mathbb{1}_{\{\alpha=1\}}+\mathbb{1}_{\{\alpha \neq 1\}} e^{-\mathrm{i} \pi \beta K(\alpha) / 2}\right)\right]\right\}$. Notice also that for any $p>0$ and $q \in \mathbf{R}$,

$$
\int_{0}^{\infty} e^{-p t} \sin (q t) t^{-(\varepsilon+1)} \mathrm{d} t=\frac{\Gamma(1-\varepsilon)}{2 \mathrm{i} \varepsilon}\left[(p+\mathrm{i} q)^{\varepsilon}-(p-\mathrm{i} q)^{\varepsilon}\right] .
$$

We deduce with a simple calculation that $S^{\alpha, \beta, \gamma, \delta} \in \mathscr{C} \mathscr{L}\left(C_{\mathrm{drf}}\right)$ implies

$$
\begin{aligned}
& d^{\eta, X}=\left(\gamma^{2}+\delta^{2}\right)^{\varepsilon / 2} \begin{cases}\sin \frac{\pi \varepsilon \beta K(\alpha)}{2} & \text { if } \quad \alpha \neq 1 \\
\sin \left(\varepsilon \arcsin \left(\frac{\delta}{\sqrt{\gamma^{2}+\delta^{2}}}\right)\right) & \text { if } \quad \alpha=1\end{cases} \\
& =\left(\gamma^{2}+\delta^{2}\right)^{\varepsilon / 2} \sin \left(\frac{\pi}{2} \varepsilon \alpha \theta(\alpha, \beta, \gamma, \delta)\right) \text {. }
\end{aligned}
$$

2) Thanks to the scaling property (2.3), and because $S^{\alpha, \beta, \gamma, \delta} \in$ $\mathscr{C} \mathscr{L}\left(C_{\mathrm{drf}}\right)$, we have for all $x>0$,

$$
\nu^{\eta, X}[x,+\infty)=\frac{\varepsilon}{\Gamma(1-\varepsilon)} \int_{0}^{\infty} \mathbf{P}\left\{S_{1}^{\alpha, \beta, \gamma, \delta}>x t^{-1 / \alpha}\right\} t^{-\varepsilon-1} \mathrm{~d} t .
$$


Since $\lim _{u \rightarrow 0,+\infty} u^{\varepsilon \alpha} \mathbf{P}\left\{S_{1}^{\alpha, \beta, \gamma, \delta}>u\right\}=0$, the change of variables $u=x t^{-1 / \alpha}$ gives

$$
\nu^{\eta, X}[x,+\infty)=\frac{1}{\Gamma(1-\varepsilon) x^{\varepsilon \alpha}} \mathbb{E}\left[\left(S_{1}^{\alpha, \beta, \gamma, \delta}\right)^{\varepsilon \alpha} \mathbb{1}_{\left\{S_{1}^{\alpha, \beta, \gamma, \delta}>0\right\}}\right] .
$$

Notice that $\mathbb{E}\left[\left(S_{1}^{\alpha, \beta, \gamma, \delta}\right)^{\varepsilon \alpha} \mathbb{1}_{\left\{S_{1}^{\alpha, \beta, \gamma, \delta}>0\right\}}\right]$ is given in (4.6). Using $S^{\alpha, \beta, \gamma, \delta} \stackrel{\mathscr{L}}{=}$ $-S^{\alpha,-\beta, \gamma,-\delta}$ and doing exactly the same calculation, we obtain for $x<0$ the same expression for $\nu^{\eta, X}(-\infty, x]$ with $(x, \beta, \delta)$ replaced by $(-x,-\beta,-\delta)$ in the right-hand side of the previous equality. Finally,

$$
\nu^{\eta, X}(\mathrm{~d} x)=\left(C(\alpha, \beta, \gamma, \delta, \varepsilon) \mathbb{1}_{\{x>0\}}+C(\alpha,-\beta, \gamma,-\delta, \varepsilon) \mathbf{1}_{\{x<0\}}\right)|x|^{-(\varepsilon \alpha+1)} \mathrm{d} x,
$$

where

$$
C(\alpha, \beta, \gamma, \delta, \varepsilon)=\frac{\left(\gamma^{2}+\delta^{2}\right)^{\varepsilon / 2}}{\pi} \Gamma(\varepsilon \alpha+1) \sin \left(\frac{\pi}{2} \varepsilon \alpha(1+\theta(\alpha, \beta, \gamma, \delta)) .\right.
$$

3) Notice that $\theta(\alpha, \beta, \gamma, \delta)=-\theta(\alpha,-\beta, \gamma,-\delta)$. Now take $\alpha^{\prime}=\varepsilon \alpha$ and $\beta^{\prime}, \gamma^{\prime}$ as in Definition $1(\mathrm{i})$, we see that $\theta(\alpha, \beta, \gamma, \delta)=\theta\left(\alpha^{\prime}, \beta^{\prime}, \gamma^{\prime}, 0\right)$, and (4.2), (4.3) imply that $\left(d^{\eta, X}, 0, \nu^{\eta, X}\right)$ corresponds to the characteristics of a stable process $S^{\alpha^{\prime}, \beta, \gamma^{\prime}} \in \mathscr{C} \mathscr{L}\left(C_{\text {skw }}\right)$. We can now conclude that a version of the Lévy process with characteristics $D^{\eta}\left(d^{X}, c^{X}, \nu^{X}\right)+\left(d^{\eta, X}, 0, \nu^{\eta, X}\right)$ is the process $d^{1 / \alpha} S^{\alpha, \beta, \gamma, \delta}+S^{\alpha^{\prime}, \beta^{\prime}, \gamma^{\prime}}$ with $S^{\alpha, \beta, \gamma, \delta}$ and $S^{\alpha^{\prime}, \beta^{\prime}, \gamma^{\prime}}$ independent. One concludes with the uniqueness of the martingale problem by Theorem III.2.16 [5] in order to show (3.2).

4) In order to show (3.3), we only need to calculate the characteristics of the sum $S^{\alpha^{\prime}, \beta^{\prime}, \gamma^{\prime}}+d^{1 / \alpha} S^{\alpha, \beta, \gamma, \delta}$, where $S^{\alpha^{\prime}, \beta^{\prime}, \gamma^{\prime}}$ and $S^{\alpha^{\prime}, \beta^{\prime}, \gamma^{\prime}}$ are independent and belong, respectively, to $\mathscr{C} \mathscr{L}\left(C_{\text {skw }}\right)$ and to $\mathscr{C} \mathscr{L}\left(D_{\alpha^{\prime}, \beta^{\prime}, \gamma^{\prime}}^{\dagger}\right)$. Actually we just need to show that the characteristics are equal to $D^{\eta}\left(d^{X}, c^{X}, \nu^{X}\right)+$ $\left(d^{\eta, X}, 0, \nu^{\eta, X}\right)$ already calculated above in 1) and 2). But this is an easy problem, since $\left(\alpha^{\prime}, \beta^{\prime}, \gamma^{\prime}, 0\right) \in C_{\text {skw }}$ and $(\alpha, \beta, \gamma, \delta) \in D_{\alpha^{\prime}, \beta^{\prime}, \gamma^{\prime}}^{\uparrow}$ is equivalent to $(\alpha, \beta, \gamma, \delta) \in C_{\mathrm{drf}}$ and $\left(\alpha^{\prime}, \beta^{\prime}, \gamma^{\prime}, 0\right) \in D_{\alpha, \beta, \gamma, \delta}^{\downarrow}$. Theorem 1 is proved.

3.2. The mixing procedure. In this subsection, we will investigate how much we can extend the identity (3.3). More precisely, taking for $1 \leqslant k \leqslant n, n$ independent processes $S^{a_{k}, b_{k}, c_{k}, d_{k}} \in \mathscr{C} \mathscr{L}(B)$, we will give weaker conditions on these coefficients in order to show identities of the type (3.3) holding with some special kind of processes which we will call Mixed Stable Processes.

For this purpose, we need some framework. Change the notation of the initial stochastic basis by $\left(\Omega^{1}, \mathscr{D}^{1},\left(\mathscr{F}_{t}^{1}\right)_{t \geqslant 0}, \mathbf{P}^{1}\right)$ and assume that $\left(\mathscr{F}_{t}^{1}\right)_{t \geqslant 0}$ is right-continuous and $\mathscr{F}_{0}^{1}$ is the trivial sub- $\sigma$-field of $\mathscr{D}^{1}$. Let $\left(\Omega^{2}, \mathscr{D}^{2},\left(\mathscr{F}_{t}^{2}\right)_{t \geqslant 0}, \mathbf{P}^{2}\right)$ be another filtered probability space; define the extension $\Omega=\Omega^{1} \times \Omega^{2}$, the tensorial product $\mathscr{D}=\mathscr{D}^{1} \otimes \mathscr{D}^{2}$ and fix a truncation function $h$.

1) Let $\Theta \subset \mathbf{R}^{d}$. For all $\xi \in \Theta, \mathbf{P}^{1, \xi}$ is the probability law on $\Omega^{1}$ of a Lévy process $S^{\xi}$ having characteristics $\left(D^{\xi}, B^{\xi}, \nu^{\xi}\right)$ measurable in $\xi$. Let a random 
variable $\theta=\left(\theta_{1}, \ldots, \theta_{d}\right) \in \Theta$ be given on $\Omega^{2}$. Take $\mathscr{F}_{t}=\bigcap_{s>t} \mathscr{F}_{s}^{1} \otimes \sigma(\theta)$ and $\mathbf{P}\left(\mathrm{d} \omega_{1}, \mathrm{~d} \omega_{2}\right)=\mathbf{P}^{2}\left(\mathrm{~d} \omega_{2}\right) \mathbf{P}^{1, \theta\left(\omega_{2}\right)}\left(\mathrm{d} \omega_{1}\right)$. Now introduce on $\Omega$ a real-valued process denoted by $M^{\theta}$, which is càdlàg, $\left(\mathscr{F}_{t}\right)_{t \geqslant 0}$-adapted, such that $M_{0}^{\theta}=0$ and for $0 \leqslant s \leqslant t$, the variable $M_{t}^{\theta}-M_{s}^{\theta}$ and the $\sigma$-field $\mathscr{F}_{s}$ are conditionally independent with respect to $\mathscr{F}_{0}$, and characterized, for all $u \in \mathbf{R}$ by

$$
\begin{aligned}
& G(u)_{s, t}=\mathbb{E}\left[\exp \left\{\mathrm{i} u\left(M_{t}^{\theta}-M_{s}^{\theta}\right)\right\} \mid \mathscr{F}_{0}\right] \\
&=\exp \left[( t - s ) \left\{\mathrm{i} u D^{\theta}-\frac{1}{2}\left(B^{\theta} u\right)^{2}\right.\right. \\
&\left.\left.-\int_{\mathbf{R}}\left(e^{\mathrm{i} u x}-1-\mathrm{i} h(x) u\right) \nu^{\theta}(\mathrm{d} x)\right\}\right] .
\end{aligned}
$$

The processes $G(u)_{\bullet, t}$ has the form II.6.7 expressed in [5], and actually, we just built a process $M^{\theta}$ which is called a process with $\mathscr{F}_{0}$-conditionally stationary independent increments (see [5, Definition II.6.2]). According to [5, Theorem II.6.5], it is a semimartingale. From now on, we will shortly call such a process an $\mathscr{F}_{0}$-Lévy process.

2) Another way of mixing is time changing. Let $\mathscr{S}$ be a Lévy process on $\Omega^{1}$ with characteristics $(D, B, \nu)$, and let $\zeta$ be an increasing $\mathbf{R}_{+}$-valued process on $\Omega^{2}$, null in 0 , without fixed times of discontinuity. Take on $\Omega$ the extension of $S$ and $\zeta$, and denote them by $M$ and $\eta$. Build $\mathbf{P}=\mathbf{P}_{1} \otimes \mathbf{P}_{2}$ and $\mathscr{F}_{t}=\bigcap_{s>t} \sigma\left(M_{r}, r \leqslant \eta_{s}\right) \vee \sigma\left(\eta_{r}, r \geqslant 0\right)$. Now introduce on $\Omega$ the process $M_{\eta}=M \circ \eta$, which is càdlàg, $\left(\mathscr{F}_{t}\right)_{t \geqslant 0}$-adapted, such that $M_{\eta_{0}}=0$ and for $0 \leqslant s \leqslant t$, the variable $M_{\eta_{t}}-M_{\eta_{s}}$ and the $\sigma$-field $\mathscr{F}_{s}$ are conditionally independent with respect to $\mathscr{F}_{0}$, and characterized, for all $u \in \mathbf{R}$ by

$$
\begin{aligned}
G(u)_{s, t} & =\mathbb{E}\left[\exp \left\{\mathrm{i} u\left(M_{\eta_{t}}-M_{\eta_{s}}\right)\right\} \mid \mathscr{F}_{0}\right] \\
& =\exp \left[\left(\eta_{t}-\eta_{s}\right)\left\{\mathrm{i} u D-\frac{1}{2}(B u)^{2}-\int_{\mathbf{R}}\left(e^{\mathrm{i} u x}-1-\mathrm{i} h(x) u\right) \nu(\mathrm{d} x)\right\}\right] .
\end{aligned}
$$

Because $\eta$ has no fixed times of discontinuity, the processes $G(u)_{., t}$ have also the form II.6.7 [5], and $M_{\eta}$ is a process with $\mathscr{F}_{0}$-conditionally independent increments and a semimartingale. As a matter of fact, Theorem 2 uses, without saying it, the following considerations. Assume $M_{\eta_{t}}-M_{\eta_{s}}$ and $\mathscr{F}_{s}$ are independent (not only $\mathscr{F}_{0}$-conditionally independent) and $\eta$ is a subordinator, then, according to Theorem $2, M_{\eta}$ is a Lévy process.

3) One, of course, can build a doubly mixed process $M_{\eta}^{\theta}$, and can separate independently $\eta$ and $\theta$ (or the components of $\theta$ as well) in the following sense: build before the processes $S_{\eta}^{\xi}$ on $\Omega$, extend once more $\Omega$, and mix on the bigger space by $\theta$ to obtain $M_{\eta}^{\theta}$.

4) From now on, when we want to express some independence between variables or processes, it is understood that we are on a filtered probability space $\left(\Omega, \mathscr{D},\left(\mathscr{F}_{t}\right)_{t \geqslant 0}, \mathbf{P}\right)$ large enough. Having understood the mixing procedure (3.4), we are able to mix stable processes. We propose the following way which turns out to be sufficient for the kind of results we are looking for. 
Fix $E \subseteq B, n \in \mathbf{N}^{*}, d=3 n, \alpha \in(0,2]$, and $h(x)=\sin x$. Consider $n$ independent stable processes $S^{\alpha, u_{k}, v_{k}, w_{k}} \in \mathscr{C} \mathscr{L}(E)$. Take $S^{\xi}=$ $\sum_{k=1}^{n} S^{\alpha, u_{k}, v_{k}, w_{k}}$ and $\theta=\left(\beta_{1}, \gamma_{1}, \delta_{1}, \ldots, \beta_{n}, \gamma_{n}, \delta_{n}\right)$ a random vector in $\mathbf{R}^{3 n}$, distributed according to a law $p^{\theta}$, such that $\left(\alpha, \beta_{k}, \gamma_{k}, \delta_{k}\right) \in E$ a.s., and introduce the corresponding $\mathscr{F}_{0}$-Lévy process $M^{\theta}$. A version is given for all $t \geqslant 0, \omega \in \Omega$, by

$$
M_{t}^{\theta}(\omega)=\sum_{k=1}^{n} S_{t}^{\alpha, \beta_{k}(\omega), \gamma_{k}(\omega), \delta_{k}(\omega)}(\omega) \quad \text { for } \quad \mathscr{F}_{0}=\bar{\sigma}_{n}:=\bigvee_{k=1}^{n} \sigma\left(\beta_{k}, \gamma_{k}, \delta_{k}\right)
$$

and is distributed by this way: for any bounded measurable function $f$,

$$
\begin{aligned}
& \mathbb{E}\left[f\left(M_{t}^{\theta}\right)\right] \\
& \quad=\int \mathbb{E}\left[f\left(\sum_{k=1}^{n} S_{t}^{\alpha, u_{k}, v_{k}, w_{k}}\right)\right] p^{\theta}\left(\mathrm{d} u_{1}, \mathrm{~d} v_{1}, \mathrm{~d} w_{1}, \ldots, \mathrm{d} u_{n}, \mathrm{~d} v_{n}, \mathrm{~d} w_{n}\right),
\end{aligned}
$$

and the random variable $\sum_{k=1}^{n} S_{t}^{\alpha, u_{k}, v_{k}, w_{k}}$ in the right-hand side of (3.6) is understood as a sum of independent stable variables. Now, in virtue of (3.6), when $n=1, \theta=(\beta, \gamma, \delta)$, it is natural to call $M^{\theta}$ a Mixed Stable Process and to denote from now on,

$$
M^{\theta}:=M^{\alpha, \beta, \gamma, \delta} \in \mathscr{M} \mathscr{C} \mathscr{L}(E) \quad \text { if } \quad(\alpha, \beta, \gamma, \delta) \in E \quad \text { a.s. }
$$

The letter $M$ stands for mixed. When we write $S$ instead of $M$, it is obviously understood, that the coefficients are deterministic, and the same rules of omissions on the coefficients for $S$ hold in $M$. One, of course, can also study mixed stable processes on their stability coefficients, but we will not be interested in this case, and we stress that in all the following, the stability coefficients are deterministic. Our aim is to clear the fact that for $n>1$, $M^{\theta}$ is also a Mixed Stable Process and that it keeps the usual properties of the proper stable processes. For example, if $\beta$ is deterministic and $\delta=0$, then the variable $M_{1}^{\alpha, \beta, \gamma}$ is distributed as $\gamma^{1 / \alpha} M_{1}^{\alpha, \beta}$, we will show that $M^{\theta}$ has similar properties.

$\mathrm{D}$ ef in ition 2 (The mixing functions). For $u, v \geqslant 0$ and $\theta_{1}, \theta_{2} \in$ $[-1,1]$, define according to the values of $\alpha \in(0,2]$ :

$$
\begin{aligned}
T_{\alpha}\left(\theta_{1}, \theta_{2}\right) & :=\mathbb{1}_{\{\alpha=1\}}+\mathbb{1}_{\{\alpha \neq 1\}} \cos \left(\frac{\pi}{2}\left(\theta_{1}-\theta_{2}\right) K(\alpha)\right), \\
g_{\alpha}\left(u, v, \theta_{1}, \theta_{2}\right) & :=\left[u^{2}+v^{2}+2 T_{\alpha}\left(\theta_{1}, \theta_{2}\right) u v\right]^{1 / 2}, \\
b_{\alpha}\left(u, v, \theta_{1}, \theta_{2}\right) & := \begin{cases}\frac{2}{\pi K(\alpha)} \arcsin \left(\frac{u \sin \left(\pi \theta_{1} K(\alpha) / 2\right)+v \sin \left(\pi \theta_{2} K(\alpha) / 2\right)}{g_{\alpha}\left(u, v, \theta_{1}, \theta_{2}\right)}\right) \\
\text { if } \alpha \neq 1, \\
\frac{\theta_{1} u+\theta_{2} v}{u+v} & \text { if } \alpha=1 .\end{cases}
\end{aligned}
$$


We present a result, used in Theorem 3 below, and which generalizes the property 2.3 on page 61 in [11] dealing with sums of independent stable variables having the same coefficient of stability and deterministic skewness, scale, and drift coefficients.

Proposition 2. Let $M^{\theta}$ be a $\bar{\sigma}_{n}$-Lévy process given by (3.5). Assume $\left(\alpha, \beta_{k}, \gamma_{k}, \delta_{k}\right) \in E$ a.s. for $E=B, C, C_{\text {skw }}, C_{\text {drf }}, S_{\text {ext }}$ or $S_{u b o}$. Define, for $1 \leqslant k \leqslant n-1$, the random variables $\left(\bar{\beta}_{1}, \bar{\gamma}_{1}, \bar{\delta}_{1}\right):=\left(\beta_{1}, \gamma_{1}, \delta_{1}\right)$ and

$\left(\bar{\beta}_{k+1}, \bar{\gamma}_{k+1}, \bar{\delta}_{k+1}\right):=\left(b_{\alpha}\left(\gamma_{k+1}, \bar{\gamma}_{k}, \beta_{k+1}, \bar{\beta}_{k}\right), g_{\alpha}\left(\gamma_{k+1}, \bar{\gamma}_{k}, \beta_{k+1}, \bar{\beta}_{k}\right), \sum_{j=1}^{k+1} \delta_{j}\right)$.

Then we have the following:

1) For all $n \in \mathbf{N}^{*}, M^{\theta}$ is a mixed stable process in the following sense: for almost all $\omega$, one has

$$
\begin{gathered}
\left(\alpha, \bar{\beta}_{n}(\omega), \bar{\gamma}_{n}(\omega), \bar{\delta}_{n}(\omega)\right) \in E, \\
\min _{1 \leqslant k \leqslant n} \beta_{k}(\omega)=\beta_{n_{\wedge}}(\omega) \leqslant \bar{\beta}_{n}(\omega) \leqslant \beta_{n_{\vee}}(\omega)=\max _{1 \leqslant k \leqslant n} \beta_{k}(\omega), \\
T_{\alpha}\left(\frac{\beta_{n_{\vee}}(\omega)}{2}, \frac{\beta_{n_{\curlywedge}}(\omega)}{2}\right) \sum_{k=1}^{n} \gamma_{k}(\omega) \leqslant \bar{\gamma}_{n}(\omega) \leqslant \sum_{k=1}^{n} \gamma_{k}(\omega),
\end{gathered}
$$

and

$$
M^{\theta} \stackrel{\mathscr{L}}{=} M^{\alpha, \bar{\beta}_{n}, \bar{\gamma}_{n}, \bar{\delta}_{n}}, .
$$

2) For all $t>0$, define $\bar{d}(\alpha, t, n)=t\left(\bar{\delta}_{n}+\mathbb{1}_{\{\alpha=1\}} 2 \pi^{-1} \bar{\beta}_{n} \bar{\gamma}_{n} \ln \left(t \bar{\gamma}_{n}\right)\right)$. Furthermore, the following scaling properties are true:

$$
\begin{aligned}
& M_{t}^{\alpha, \bar{\beta}_{n}, \bar{\gamma}_{n}, \bar{\delta}_{n}} \stackrel{\stackrel{d}{=}}{=} M_{1}^{\alpha, \bar{\beta}_{n}, \bar{\gamma}_{n} t, \bar{\delta}_{n} t} \stackrel{d}{=} M^{\alpha, \bar{\beta}_{n}, 1, \bar{\delta}_{n} / \bar{\gamma}_{n}} \circ\left(\bar{\gamma}_{n} t\right) \\
& \stackrel{d}{=} \bar{d}(\alpha, t, n)+\left(\bar{\gamma}_{n} t\right)^{1 / \alpha} M_{1}^{\alpha, \bar{\beta}_{n}} .
\end{aligned}
$$

With $\rho$ defined in (3.1), one has

$\mathbf{P}\left\{M_{t}^{\alpha, \bar{\beta}_{n}, \bar{\gamma}_{n}, \bar{\delta}_{n}}>0\right\}=\mathbb{E}\left[\rho\left(\alpha, \bar{\beta}_{n}, \bar{\gamma}_{n}, \bar{\delta}_{n}\right)\right] \quad$ if $\quad M^{\alpha, \bar{\beta}_{n}, \bar{\gamma}_{n}, \bar{\delta}_{n}} \in \mathscr{M} \mathscr{C} \mathscr{L}\left(C_{\text {drf }}\right)$.

$\mathrm{R}$ e m a r k 4. What is really important in the construction of $M^{\theta}$, is that no independence between the $\beta_{i}, \gamma_{j}, \delta_{k}$ is required. Consider, for example, the case $n=2$. Notice that $\beta_{1}=\beta_{2}$ implies $\bar{\gamma}_{2}=\gamma_{1}+\gamma_{2}$ and $\bar{\beta}_{2}=\beta_{1}=\beta_{2}$, and that $\gamma_{1}=\gamma_{2}$ implies $\bar{\gamma}_{2}=2, \gamma_{1} T_{\alpha}\left(\beta_{1} / 2, \beta_{2} / 2\right)$ and $\bar{\beta}_{2}=$ $\left(\beta_{1}+\beta_{2}\right) / 2$. Now, take $S^{\alpha, 0} \in \mathscr{C} \mathscr{L}\left(C_{\text {skw }}\right)$ and $S^{\varepsilon} \in \mathscr{C} \mathscr{L}\left(S_{u b o}\right)$ independent. On the other hand, let $\beta$ be deterministic, two independent processes $S^{\alpha, \beta}$, $S^{\alpha,-\beta} \in \mathscr{C} \mathscr{L}(B)$ and $\left(\gamma_{1}, \gamma_{2}\right)$ independent from $\left(S^{\alpha, \beta}, S^{\alpha,-\beta}\right)$, and $\gamma_{1}, \gamma_{2}$ distributed as $S_{1}^{\varepsilon}$. According to Proposition 2, and then Theorem 1, one obtains immediately: if $\gamma_{1}=\gamma_{2}$ or if $\gamma_{1}$ is independent from $\gamma_{2}$, then $S^{\alpha, \beta} \circ \gamma_{1}+S^{\alpha,-\beta} \circ \gamma_{2} \stackrel{d}{=} M_{1}^{\alpha, 0,2 T_{\alpha}(\beta, 0) S_{1}^{\varepsilon} \stackrel{d}{=}} S^{\alpha, 0} \circ\left(2 T_{\alpha}(\beta, 0) S_{1}^{\varepsilon}\right) \stackrel{d}{=} S_{1}^{\varepsilon \alpha, 0,2 T_{\alpha}(\beta, 0)}$. 
This relation can be explained by $S^{\alpha, \beta}+S^{\alpha,-\beta} \stackrel{\mathscr{L}}{=} S^{\alpha, 0,2} T_{\alpha}(\beta, 0)$. Notice also that this result can be expressed similarly with $\beta$ a r.v. not necessarily independent from $\gamma_{1}, \gamma_{2}$.

Proof of Proposition 2. It is a simple complex analysis argument.

1) For $0 \leqslant s \leqslant t, u \in \mathbf{R}$, let $G(u)_{s, t}=\mathbb{E}\left[\exp \left\{\mathrm{i} u\left(M_{t}^{\theta}-M_{s}^{\theta}\right)\right\} \mid \bar{\sigma}_{n}\right]$. Considering only the case $\alpha \neq 1$, (the case $\alpha=1$ being obvious), a version is given, according to (2.2), (3.4), (3.5), by

$$
G(u)_{s, t}=\exp \left\{(t-s) \sum_{k=1}^{n} \mathrm{i} u \delta_{k}-\gamma_{k}|u|^{\alpha} e^{-\mathrm{i} \pi \beta_{k} K(\alpha) \operatorname{sign}(u) / 2}\right\} .
$$

Let $\bar{\delta}_{n}:=\sum_{k=1}^{n} \delta_{k}, Z_{k}:=\gamma_{k} e^{-\mathrm{i} \pi \beta_{k} K(\alpha) / 2}, \bar{Z}_{n}:=\sum_{k=1}^{n} Z_{k}$, let $\widetilde{Z}_{n}$ denote the complex conjugate of $\bar{Z}_{n}$, and let $\bar{\gamma}_{n}:=\left|\bar{Z}_{n}\right|$ and $\bar{\beta}_{n}:=-2 \arg \left(\bar{Z}_{n}\right) /(\pi K(\alpha))$. Then we have the representation

$$
G(u)_{s, t}=\exp \left\{(t-s)\left(\mathrm{i} u \bar{\delta}_{n}-|u|^{\alpha}\left(\bar{Z}_{n} \mathbb{1}_{\{u \geqslant 0\}}+\widetilde{Z}_{n} \mathbb{1}_{\{u \leqslant 0\}}\right)\right)\right\} .
$$

A simple calculation gives $\left(\bar{\beta}_{2}, \bar{\gamma}_{2}\right)=\left(b_{\alpha}, g_{\alpha}\right)\left(\gamma_{1}, \gamma_{2}, \beta_{1}, \beta_{2}\right)$, and generally, the expression of $\left(\bar{\beta}_{n}, \bar{\gamma}_{n}\right)$ follows by recurrence. Finally, with definition (3.4), one can write for all $u \in \mathbf{R}, 0 \leqslant s \leqslant t$,

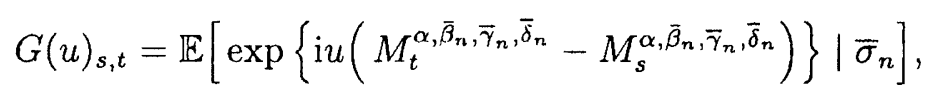

and (3.9) is true in virtue of the uniqueness of the martingale problem, [5, Theorem II.6.11]. Now, use the scaling property (2.3) and the mixing procedure (3.6), then it is easy to see, for example, that

$$
\begin{aligned}
G(u)_{0, t} & \stackrel{d}{=} \mathbb{E}\left[\exp \left\{\mathrm{i} u\left(\bar{\delta}_{n} t+M^{\alpha, \bar{\beta}_{n}} \circ\left(\bar{\gamma}_{n} t\right)\right)\right\} \mid \bar{\sigma}_{n}\right] \\
& \stackrel{d}{=} \mathbb{E}\left[\exp \left\{\mathrm{i} u\left(\bar{\delta}_{n} t+\left(\bar{\gamma}_{n} t\right)^{1 / \alpha} M_{1}^{\alpha, \bar{\beta}_{n}}\right)\right\} \mid \bar{\sigma}_{n}\right]
\end{aligned}
$$

and get (3.10) because $\mathbb{E}\left[\exp \left\{\mathrm{iu} M_{t}^{\alpha, \bar{\beta}_{n}, \bar{\gamma}_{n}, \bar{\delta}_{n}}\right\}\right]=\mathbb{E}\left[G(u)_{0, t}\right]$. The rest is obtained similarly.

2) It is immediate that $\bar{\delta}_{n}=0$ a.s. (respectively, $\geqslant 0$ ) if a.s. each $\left(\alpha, \beta_{k}, \gamma_{k}, \delta_{k}\right) \in C_{\mathrm{drf}}$ (respectively, $\left.S_{u b o}\right)$. Since $\left|\arg \left(Z_{k}\right)\right|<\pi / 2$ a.s., we have $\left|\arg \left(\bar{Z}_{n}\right)\right|<\pi / 2$ a.s., and this obviously gives $\left|\bar{\beta}_{n}\right| \leqslant \max \left\{\left|\beta_{k}\right|, 1 \leqslant k \leqslant n\right\}$. This ensures that $\left(\alpha, \bar{\beta}_{n}, \bar{\gamma}_{n}, \bar{\delta}_{n}\right) \in B$ (respectively, $\left.C, C_{\mathrm{drf}}, C_{\mathrm{skw}}\right)$ a.s., if a.s. each $\left(\alpha, \beta_{k}, \gamma_{k}, \delta_{k}\right) \in B$ (respectively, $\left.C, C_{\text {drf }}, C_{\text {skw }}\right)$ a.s. When each $Z_{k}$ is in the same semi-axis, i.e., $\left(\alpha, \beta_{k}, \gamma_{k}, \delta_{k}\right) \in S_{\text {ext }}$ (respectively, $\left.S_{u b o}\right)$ a.s., then $\left(\alpha, \bar{\beta}_{n}, \bar{\gamma}_{n}, \bar{\delta}_{n}\right) \in S_{\text {ext }}$ (respectively, $\left.S_{u b o}\right)$ a.s.

3) It remains to give the precise upper and lower bounds for $\bar{\beta}_{n}$ and $\bar{\gamma}_{n}$. When $\alpha=1, \bar{\gamma}_{n}=\sum_{k=1}^{n} \gamma_{k}, \bar{\beta}_{n}$ is a convex combination of the $\beta_{k}$ and everything is clear. Otherwise, write

$$
\frac{\bar{Z}_{n}}{\sum_{k=1}^{n}\left|Z_{k}\right|}=\sum_{k=1}^{n} \frac{\left|Z_{k}\right|}{\sum_{k=1}^{n}\left|Z_{k}\right|} e^{\mathrm{i} \arg \left(Z_{k}\right)}
$$


Then we see that $\bar{Z}_{n} / \sum_{k=1}^{n}\left|Z_{k}\right|$ is in the convex envelope contained in the right half of the unit disk and having summits at the complex points $e^{\operatorname{iarg}\left(Z_{k}\right)}$. If we denote $n_{\wedge}=\arg \min \left\{\beta_{k}, 1 \leqslant k \leqslant n\right\}$ and $n_{\vee}=\arg \max \left\{\beta_{k}, 1 \leqslant k \leqslant n\right\}$, then $\bar{\beta}_{n}=-2 \arg \left(\bar{Z}_{n} / \sum_{k=1}^{n}\left|Z_{k}\right|\right) /(\pi K(\alpha))$ satisfies

$$
\begin{aligned}
\min _{1 \leqslant k \leqslant n}\left(-\frac{\pi}{2} \beta_{k} K(\alpha)\right) & \leqslant-\frac{\pi}{2} \bar{\beta}_{n} K(\alpha) \\
& \leqslant \max _{1 \leqslant k \leqslant n}\left(-\frac{\pi}{2} \beta_{k} K(\alpha)\right) \Longleftrightarrow \beta_{n_{\wedge}} \leqslant \bar{\beta}_{n} \leqslant \beta_{n_{\vee}} .
\end{aligned}
$$

We also see that $\bar{Z}_{n} / \sum_{k=1}^{n}\left|Z_{k}\right|$ is situated in the outer isosceles triangle having summits at $0, e^{\operatorname{iarg}\left(Z_{n \wedge}\right)}$, and $e^{\operatorname{iarg}\left(Z_{n \vee}\right)}$. This implies that $\left|\bar{Z}_{n}\right| / \sum_{k=1}^{n}\left|Z_{k}\right|$ is bigger than the length of the main height of this triangle, i.e.,

$$
\begin{aligned}
\cos \left(\frac{\pi}{4}\left(\beta_{n_{\curlywedge}}-\beta_{n_{\curlywedge}}\right) K(\alpha)\right) & =\cos \left(\frac{1}{2}\left[\arg \left(Z_{n_{\vee}}\right)-\arg \left(Z_{n_{\curlywedge}}\right)\right]\right) \\
& \leqslant\left|\bar{Z}_{n}\right|\left(\sum_{k=1}^{n}\left|Z_{k}\right|\right)^{-1} \leqslant 1 .
\end{aligned}
$$

Proposition 2 is proved.

Now, we want to show a theorem, consequence of Theorem 1 and Proposition 2 and which furnishes identities analogous to (3.3) for the sum of independent stable process.

Preliminaries of Theorem 3. Fix $n \in \mathbf{N}^{*}$ and let $S^{a_{k}, b_{k}, c_{k}, d_{k}} \in$ $\mathscr{C} \mathscr{L}(B), 1 \leqslant k \leqslant n$, be $n$ independent processes such that $a_{1} \leqslant a_{2} \leqslant \cdots \leqslant$ $a_{n}$ and

$$
D=\bigcap_{k=1}^{n}\left[a_{k}, \frac{2}{1+\left|b_{k} \bar{K}\left(a_{k}\right)\right|}\right] \neq \varnothing .
$$

If $a_{k}>1$ and $\left|b_{k}\right|=1$, then the interval is reduced to $\left\{a_{k}\right\}$ and an immediate consequence is: $a_{l}>a_{k}>1$ and $\left(a_{l}, b_{l}, c_{l}, d_{l}\right),\left(a_{k}, b_{k}, c_{k}, d_{k}\right) \in S_{\text {ext }}$ implies $D=\varnothing$. Notice that if $D \neq \varnothing$, then $a_{n} \in D$ always holds. According to Theorem 1 , for any $\alpha \in D$, there exist $S^{\alpha, u_{k}, v_{k}, w_{k}} \in \mathscr{C} \mathscr{L}\left(D_{a_{k}, b_{k}, c_{k}}^{\uparrow}\right)$ (see Definition 1), such that

$$
S^{a_{k}, b_{k}, c_{k}, d_{k}} \stackrel{\mathscr{L}}{=} d_{k} S^{1}+S^{\alpha, u_{k}, v_{k}, w_{k}} \circ S^{a_{k} / \alpha},
$$

where each $S^{a_{k} / \alpha} \in \mathscr{C} \mathscr{L}\left(S_{u b o}\right)$ is independent from $S^{\alpha, u_{k}, v_{k}, w_{k}}$. Now choose $n$ processes $S^{\alpha, u_{k}, v_{k}, w_{k}}$ and $n$ processes $S^{a_{k} / \alpha}$ such that $S^{\alpha, u_{k}, v_{k}, w_{k}}$ and $S^{a_{k} / \alpha}$ are independent, and for $k \neq l$, the couples $\left(S^{\alpha, u_{k}, v_{k}, w_{k}}, S^{a_{k} / \alpha}\right)$ and $\left(S^{\alpha, u_{l}, v_{l}, w_{l}}, S^{a_{l} / \alpha}\right)$ are independent. Define with $T_{\alpha}$ given in (3.7),

$$
u_{n \vee}=\max _{1 \leqslant k \leqslant n} u_{k}, \quad u_{n \wedge}=\min _{1 \leqslant k \leqslant n} u_{k}, \quad \text { and } \quad U=T_{\alpha}\left(\frac{u_{n_{\vee}}}{2}, \frac{u_{n_{\wedge}}}{2}\right) .
$$

Now, for $t \geqslant 0$ and $1 \leqslant k \leqslant n-1$, build the variables $\bar{\beta}_{k}^{t}, \bar{\gamma}_{k}^{t}$, and $\bar{\delta}_{k}^{t}$ as follows: using Proposition 2, define first the variables $\beta_{k}^{t}=u_{k}, \gamma_{k}^{t}=v_{k} S_{t}^{a_{k} / \alpha}$, 
$\delta_{k}^{t}=d_{k} t+w_{k} S_{t}^{a_{k} / \alpha}$ and then, exactly as in (3.8), $\left(\bar{\beta}_{1}^{t}, \bar{\gamma}_{1}^{t}, \bar{\delta}_{1}^{t}\right)=\left(\beta_{1}^{t}, \gamma_{1}^{t}, \delta_{1}^{t}\right)$, and

$$
\begin{aligned}
& \left(\bar{\beta}_{k+1}^{t}, \bar{\gamma}_{k+1}^{t}, \bar{\delta}_{k+1}^{t}\right) \\
& \quad:=\left(b_{\alpha}\left(\gamma_{k+1}^{t}, \bar{\gamma}_{k}^{t}, \beta_{k+1}^{t}, \bar{\beta}_{k}^{t}\right), g_{\alpha}\left(\gamma_{k+1}^{t}, \bar{\gamma}_{k}^{t}, \beta_{k+1}^{t}, \bar{\beta}_{k}^{t}\right), \sum_{j=1}^{k+1} \delta_{j}^{t}\right) .
\end{aligned}
$$

Theorem 3. Let $n$ independent processes $S^{a_{k}, b_{k}, c_{k}, d_{k}} \in \mathscr{C} \mathscr{L}(B), 1 \leqslant$ $k \leqslant n$, be such that (3.11) is satisfied. Assume $\alpha \in D,\left(u_{k}, v_{k}, w_{k}\right)$ are given by (3.12) and $u_{n \vee}, u_{n \wedge}, U$ are given in (3.13). There exist processes $\bar{\beta}, \bar{\gamma}$, and $\bar{\delta}$ (whose version is given in (3.14) by $\bar{\beta}_{n}^{\bullet}, \bar{\gamma}_{n}^{\bullet}, \bar{\delta}_{n}^{*}$ ), satisfying for all $t>0$ and almost all $\omega \in \Omega:\left(\alpha, \bar{\beta}_{t}(\omega), \bar{\gamma}_{t}(\omega), \bar{\delta}_{t}(\omega)\right) \in B$,

$$
u_{n \wedge} \leqslant \bar{\beta}_{t}(\omega) \leqslant u_{n \vee}, \quad U \sum_{k=1}^{n} v_{k} S_{t}^{a_{k} / \alpha}(\omega) \leqslant \bar{\gamma}_{t}(\omega) \leqslant \sum_{k=1}^{n} v_{k} S_{t}^{a_{k} / \alpha}(\omega),
$$

and with the mixing procedure (3.6) and $\rho$ defined in (3.1), one has

$$
\sum_{k=1}^{n} S_{t}^{a_{k}, b_{k}, c_{k}, d_{k}} \stackrel{d}{=} M_{1}^{\alpha, \bar{\beta}_{t}, \bar{\gamma}_{t}, \bar{\delta}_{t}} \stackrel{d}{=}\left(\bar{\delta}_{t}+\mathbb{1}_{\{\alpha=1\}} \frac{2 \bar{\beta}_{t} \bar{\gamma}_{t}}{\pi} \ln \bar{\gamma}_{t}\right)+\left(\bar{\gamma}_{t}\right)^{1 / \alpha} M_{1}^{\alpha, \bar{\beta}_{t}}
$$

$$
\mathbf{P}\left\{M_{1}^{\alpha, \bar{\beta}_{t}, \bar{\gamma}_{t}, \bar{\delta}_{t}}>0\right\}=\mathbb{E}\left[\rho\left(\alpha, \bar{\beta}_{t}, \bar{\gamma}_{t}, \bar{\delta}_{t}\right)\right] \quad \text { if } \quad\left(\alpha, \bar{\beta}_{t}, \bar{\gamma}_{t}, \bar{\delta}_{t}\right) \in\left(C_{\mathrm{drf}}\right) \quad \text { a.s. }
$$

$\mathrm{R} \mathrm{e} \mathrm{mark} 5$. We emphasize the following: contrarily to the identity (3.3), the identity (3.15) reads for each $t \geqslant 0$, because the pro-

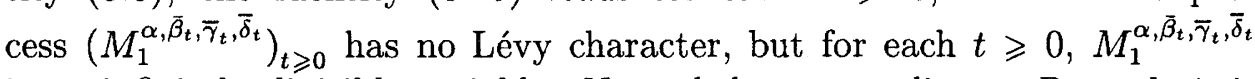
is an infinitely divisible variable. Nevertheless, according to Remark 4 , it is immediate that the identities $u_{k}=u_{1}$, for all $1 \leqslant k \leqslant n$, imply that $\bar{\beta}$ is constant equal to $u_{1}$ and $\bar{\gamma}$ is equal to the subordinator $\sum_{k=1}^{n} v_{k} S^{a_{k} / \alpha}$. Moreover, assume $u_{1}=0$ (which is equivalent to $b_{k}=0$, for all $1 \leqslant k \leqslant n$ ), then it is better to write the identity in law with a time changed Brownian motion.

$\mathrm{Pr}$ o of of $\mathrm{Th}$ e ore $\mathrm{m} 3$. It is an immediate consequence of the previous developments. By virtue of Theorem 1, one has

$$
\sum_{k=1}^{n} S^{a_{k}, b_{k}, c_{k}, d_{k}} \stackrel{\mathscr{L}}{=} \sum_{k=1}^{n} d_{k} S^{1}+\sum_{k=1}^{n} S^{\alpha, u_{k}, v_{k}, w_{k}} \circ S^{a_{k} / \alpha},
$$

where $S^{a_{k} / \alpha}$ and $S^{\alpha, u_{k}, v_{k}, w_{k}}$ are chosen as in the preliminaries of Theorem 1. According to Proposition 2, it is immediate that for each $t \geqslant 0$, a version of $\sum_{k=1}^{n} d_{k} S^{1}+S^{\alpha, u_{k}, v_{k}, w_{k}} \circ S_{t}^{a_{k} / \alpha}$ is $M_{1}^{\alpha, \bar{\beta}_{t}, \bar{\gamma}_{t}, \bar{\delta}_{t}}$, and the rest is obtained by the same means. 


\section{Appendix: Lévy character of stable processes}

Recall that a Lévy process $X$ is entirely characterized by its Lévy exponent

$$
\varphi(u)=\frac{\ln \mathbb{E}\left[e^{\mathrm{i} u X_{t}}\right]}{t}=\mathrm{i} u D-\frac{B^{2} u^{2}}{2}+\int\left[e^{\mathrm{i} u x}-1-\mathrm{i} u h(x)\right] \nu(\mathrm{d} x), \quad t>0 .
$$

The real $D$ is called drift coefficient, $B$ is a positive real called Brownian coefficient and $\nu$ is a positive measure giving no mass to 0 , called Lévy measure and integrating $x^{2} \wedge 1$. The truncation function $h$ could be any real bounded function such that $\lim _{x \rightarrow 0}(h(x)-x) / x^{2}=0$. The functional

$$
\underline{D}=\underline{D}(h, D)=D-\int h(x) \nu(\mathrm{d} x) \text { if the integral is finite, }
$$

is called displacement coefficient relative to $h$. Notice that if $B=0, \nu$ integrates $|y| \wedge 1$, and $\nu(0,+\infty)=0$ (respectively, $\nu(-\infty, 0)=0$ ), then the support of the law of $X_{t}$ is $[\underline{D} t,+\infty)$ (respectively, $\left.(-\infty, \underline{D} t]\right)$. Otherwise the law of $X_{t}$ has a support equal to $\mathbf{R}$.

Notice that if $h^{\prime}$ is another truncation function, then its corresponding drift is the finite quantity $D^{\prime}=D+\underline{D}\left(h-h^{\prime}, D\right)$. A careful examination of the proofs of Theorems C.2 and C.3 on pages 9-12 of [11] allow one to calculate the characteristics $(D, B, \nu)$ of a stable process $S^{a, b, c, d}$ when $h(x)=\sin x$ :

$$
\begin{aligned}
D & =d+c \sin \left(\frac{\pi b K(a)}{2}\right) \mathbb{1}_{\{a \neq 1\}}, \quad B=\sqrt{2 c} \mathbb{1}_{\{a=2\}}, \\
\nu(\mathrm{d} x) & =\left(\lambda_{0}(a, b, c) \mathbb{1}_{\{x>0\}}+\lambda_{0}(a,-b, c) \mathbf{1}_{\{x<0\}}\right)|x|^{-(a+1)} \mathrm{d} x \mathbb{1}_{\{a<2\}}, \\
\lambda_{0}(a, b, c) & =\frac{c}{\pi} \Gamma(a+1) \begin{cases}\sin \left(\frac{\pi}{2} a(1+\bar{K}(a) b)\right) & \text { if } a \neq 1, \\
1+b & \text { if } a=1 .\end{cases}
\end{aligned}
$$

$\mathrm{R} \mathrm{e} \mathrm{m}$ a $\mathrm{rk}$ 6. Despite the large literature devoted to stable laws, we found that these characteristics do not appear explicitly. Moreover, the stable Lévy exponents can be expressed in different ways (and, of course, the coefficients change). The form (2.2) is not the best one to recover the characteristics, but in [6], we preferred it, because it turned out to be the most useful to express some mixture properties relative to the coefficients (see Proposition 2). For stable processes, the usage is to call the coefficient $\delta$ drift, although in certain case (see (4.1), (4.2) for $a<1$ and $a=1$ ), it can correspond to a displacement coefficient. Notice also that the Lévy measure integrates $|x| \wedge 1$ if and only if $a<1$ (then the process has finite variation). It is extreme (i.e., $\lambda_{0}(a, b, c)=0$ or $\lambda_{0}(a,-b, c)=0$ ) if and only if $b=-1$ (totally skewed to the left) or $b=1$ (totally skewed to the right), and then the process has only negative jumps or positive jumps. The process is symmetrical (i.e., $\lambda_{0}(a, b, c)=\lambda_{0}(a,-b, c)$ ) if and only if $b=0$. Finally, a stable process $S^{a, b, c, d}$ is a subordinator (i.e., an increasing Lévy process) if 
and only if $a<1, b=1$, and $d \geqslant 0$. We also recall a known result (see [11, $2.2 .30,2.3 .5 \mathrm{a}$, and 2.6.20] for $t=1)$. Let $S^{a, b, c, d} \in \mathscr{C} \mathscr{L}\left(C_{\mathrm{drf}}\right), t>0$, and let $\rho$ be defined in (3.1). Then

$$
\mathbf{P}\left\{S_{t}^{a, b, c, d}>0\right\}=\rho(a, b, c, d),
$$

and for $\varepsilon<1$ if $a<1, b=1$ and otherwise $-1 / a<\varepsilon<1$, one has

$$
\begin{aligned}
\mathscr{M}_{+}^{a, b, c, d}(\varepsilon, t) & :=\mathbb{E}\left[\left(S_{t}^{a, b, c, d}\right)^{\varepsilon a} \mathbb{1}_{\left\{S_{t}^{a, b, c, d}>0\right\}}\right] \\
& =\left(t \sqrt{c^{2}+d^{2}}\right)^{\varepsilon} \frac{\sin (\pi \varepsilon a \rho)}{\sin (\pi \varepsilon a)} \frac{\Gamma(1-\varepsilon)}{\Gamma(1-\varepsilon a)} .
\end{aligned}
$$

Acknowledgements. I would like to thank Prof. R. Höpfner and J. Jacod for their helpful comments and discussions. A part of this work was done in the Laboratory of Probability of the University of Paris VI.

\section{REFERENCES}

1. Bertoin J., Doney R. A. Spitzer's conditions for random walks and Lévy processes. Ann. Inst. H. Poincaré, 1997, v. 33, № 2, p. 167-178.

2. Феллер В. Введение в теорию вероятностей и ее приложения. Т. 2. М.: Мир, 1984, $752 \mathrm{c}$.

3. Гнеденко Б.В., Колмогоров А.Н. Предельные распределения для сумм независимых случайных величин. М.-Л.: Гостехиздат, 1949, 264 с.

4. Ishikawa $Y$. On the lower bound of the density for jump processes in small time. Bull. Sci. Math., 1993, v. 117, № 4, p. 463-483.

5. Jacod J., Shiryaev A.N. Limit theorems for stochastic processes. Berlin: Springer, $1987,601 \mathrm{p}$.

6. Jedidi $W$. PHD thesis. LPMA, Université Paris VI.

7. Lévy P. Théorie des erreurs de la loi de Gauss et les lois exceptionnelles. - Bull. Soc. Math. France, 1924, v. 52, p. 49-85.

8. Picard J. Density in small time for Lévy processes. — ESAIM, Probab. Statist., 1997, v. 1, p. $357-389$.

9. Sato K.I. Lévy Processes and Infinitely Divisible Distributions. Cambridge: Cambridge Univ. Press, 1999, 486 p.

10. Золотарев B.M. Распределение суперпозиции безгранично делимых процессов. - Теория вероятн. и ее примен., 1958, т. 3, в. 2, с. 197-200.

11. Zolotarev V. M. One-Dimensional Stable Laws. Providence: Amer. Math. Soc., 1986, $284 \mathrm{p}$. 